\title{
Active manipulation of EM fields in open waveguides
}

\author{
Daniel Onofrei*and Richard Albanese ${ }^{\dagger}$
}

November 21, 2016

\section{Contents}

1 Introduction $\quad 2$

1.1 Main Question and Motivation . . . . . . . . . . . . . . . . . 2

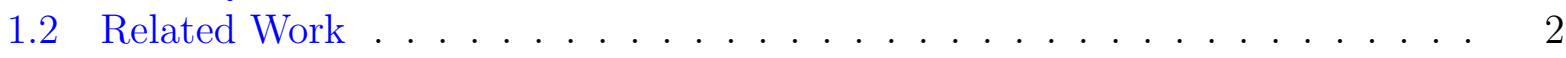

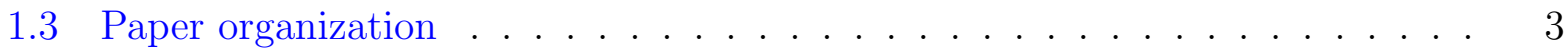

2 Theoretical results $\quad 3$

2.1 Problem Formulation . . . . . . . . . . . . . . . . . 3

2.2 Existence results . . . . . . . . . . . . . . . . . . . . . 5

3 Feasibility discussion $\quad 10$

3.1 Computation of the array currents to null the $T M_{01}$ mode . . . . . . . . . 15

3.2 Method of Moments checks on the analytical array design . . . . . . . . . . . 20

3.3 Slot antennas and arrays . . . . . . . . . . . . . . . . . . 22

4 Summary and Comments $\quad 23$

\begin{abstract}
The central question addressed in this paper is whether we can introduce an antenna array into a circular cylindrical waveguide to accurately control or determine the local field around the array with vanishingly small reflection from the boundary of the waveguide. We ask this question since we perceive the answer to be important to the applied community through its relevance for applications to electromagnetic shielding or slot antennas. We make use of the results presented in [44] for the scalar Helmholtz equation and show, by using the Debye representation in waveguides, how they apply to the current EM waveguide model. We prove the existence of a class of solutions and include a feasibility discussion for the antenna synthesis problem together with relevant simulations.
\end{abstract}

Key words. Active manipulation, waveguides, Debye potentials, layer potentials, antenna synthesis, slot antennas.

${ }^{*}$ Department of Mathematics, University of Houston, TX, e-mail: onofrei@math.uh.edu

${ }^{\dagger}$ ADED Co., San Antonio, TX, e-mail: richard.albanese2@gmail.com

(C) 2016. This manuscript version is made available under the Elsevier user license http://www.elsevier.com/open-access/userlicense/1.0/ 


\section{Introduction}

\subsection{Main Question and Motivation}

The general area of this effort concerns control of scalar or vector fields. There are several aspects of field control. On one hand, design of radiators can be addressed to provide specified beam structures in air or some other homogeneous material with particular regard, for example, for forming a prescribed spot on a target object or material. Or, the desire might be to illuminate a specific volume. This set of problems, spot size control or volume control, become far more difficult when the medium is heterogeneous with respect to propagation properties. Pioneering work concerning localization of field within compact structures by controlling tangential electric currents around the penetrable structure was done in [29, 30, 31]. Localizing fields, whether acoustic or electromagnetic, for volume energy deposition is addressed for medical applications [8, 37, 50].

A second pattern of field control involves use of a field to combine with the field from a second source to modify the second source field. Military jamming may be an example of this second pattern of field control. Active control of incoming waves by a designed source for the purpose of obscuring a defended object (active cloaking) is another example of this second pattern.

Our goal in this report is to show how small antennas may be placed within waveguides so that internal waveguide fields may be locally manipulated while maintaining arbitrary small reflections from the waveguide walls. We will show this theoretically and then discuss the feasibility of our results by focusing on the problem of locally nulling a transverse magnetic mode within a cylindrical waveguide using a small array of dipoles. Our feasibility discussion will suggest how, in principle, such field control strategy could be applied to steering beam formation by slot arrays. In more general terms we are studying how to control fields in local areas using proximate electromagnetic sources.

\subsection{Related Work}

As far as we are aware, active designs for the manipulation of fields appear to have occurred initially in the context of low-frequency acoustics (or active noise cancellation). We perceive the works of Leug [34] (feed-forward control of sound) and Olson \& May [43] (feedback control of sound) as pioneering in this field of acoustic control. The reviews [48] [35], [47], [16], [46] [13] provide detailed accounts of past and recent developments in acoustic active control. In a waveguide geometry, acoustic noise control was considered in [51] for a finite length duct, and suppression of unwanted electromagnetic modes was studied in [25] for a rectangular waveguide and respectively in [17] for slotted waveguides.

In a very interesting article, in [38] the author has outlined a shielding strategy that hides a compact object in three dimensional space using sensors and active emitters on the surface of the body. It is stated in [38] that the proposed method does not require knowledge of the incident field although we perceive that, in the electromagnetic regime, causality will imply substantial delay for shielding of larger areas. The work in [38] is computationally supported through a scalar (acoustic) example. In [11], [12], with the assumption of foreknowledge of the interrogating field, the authors have experimentally demonstrated that a cylinder can 
be protected from unwanted interrogation by using a surface array of antennas. Further experimental results on this type of active protection are presented in [36] for quasistatic regime and in [10] for finite frequency regime.

A parallel effort on the problem of active protection from unwanted interrogation is presented in [20, 21, 22, 18, 19] (see also [42] and [55] for further numerical explorations). This scheme uses exterior active sources (antennas) to manipulate the fields. This approach for 2D quasistatics was introduced in [20] and mathematically analyzed in [19]. The high frequency scalar case was studied in the last section of [20] and in [21, 22] (see also [18] for a recent review) and three active sources (antennas) were sufficient to create a zero field region in the interior of their convex hull while producing a vanishingly small field in the far field. The broadband character of the proposed scheme was numerically observed in [21]. In [42], the authors provide evidence that increasing the number of antennas to null the field around an object will reduce the overall power used by the total system. In the limit when infinitely many sources are used one obtains the interior active scheme proposed in [38].

One of the authors of the present paper (Onofrei) has developed a general approach, based on the theory of boundary layer potentials [45], for the active manipulation of quasi-static fields with just one active source (antenna) in three dimensions. This approach provides a compact quiet zone exterior to the active source. These ideas were later extended to the scalar Helmholtz equation in [44].

\subsection{Paper organization}

In this paper, we extend the scalar results in [44] to the (vector) electromagnetic case to locally control $T M$ or $T E$ modes in an open circular waveguide. We conclude the paper with a feasibility study using computation to illustrate the effects of a small array in a perfectly conductive circular wave guide.

The paper is organized as follows: In Section 2 we introduce the main problem of local nulling a waveguide TM or TE mode (Question 1) and the prove the existence of a class of solutions for Question 1. Our mathematical development indicates that local control of fields within circular cylindrical waveguides can be achieved by a combination of electric and magnetic currents. Section 3 presents a feasibility discussion for a twenty four element array which seems to successfully control the longitudinal component of $T M$ fields near its structure. An indication of possible applications to slot antennas and slot arrays is presented in the last part of this section. The final section provides summary and conclusions.

\section{Theoretical results}

\subsection{Problem Formulation}

We consider a circular cylindrical waveguide of radius $R$ filled with air and with perfectly conducting walls. Thus we consider the waveguide $D=(-\infty,+\infty) \times B_{R}(\mathbf{0})$, where, in what follows, $B_{R}(\mathbf{p})$ is the disc centred in $\mathbf{p}$ situated in a plane perpendicular to the $O x$ axis (the axis of the waveguide). 
Consider a finite length, electromagnetically transparent support structure in the center of the waveguide on which a vanishingly thin antenna is etched (Figure 1 presents a sketch for the geometry for an antenna with straight edges). Let $D_{a} \Subset D$, (for some sufficiently small constant $\zeta$ ), smooth compact domain of $\mathbb{R}^{3}$, denote the support of this thin antenna etched on the axially symmetric transparent shell. The region of interest (control region) will be denoted by $D_{c}$, and it will be assumed to be a bounded smooth open connected set with $D_{c} \Subset D \backslash \bar{D}_{a}$ where here and in what follows $\Subset$ denotes a compact embedding. We denote by $\left(\mathbf{E}_{i}, \mathbf{B}_{i}\right)$ an arbitrary TM or TE field propagating in the waveguide. We denote by $\left(\mathbf{E}_{s}, \mathbf{B}_{s}\right)$ the electromagnetic field produced by the antenna.

The general question we want to study is:

Question 1. Can we synthesize an active source (antenna) $D_{a}$ placed inside the waveguide $D$ such that the field of this antenna $\left(\mathbf{E}_{s}, \mathbf{B}_{s}\right)$, has the property that in the region of interest $D_{c},\left(\mathbf{E}_{s}, \mathbf{B}_{s}\right)$ almost cancels $\left(\mathbf{E}_{i}, \mathbf{B}_{i}\right)$ for $T M$ or $T E$ respectively while producing vanishingly small reflected fields from the waveguide walls?

We will show that antenna currents can be defined so that the waveguide field is nulled near the antenna, and, the antenna fields will vanishingly perturb the field at or near the wall of the waveguide thus producing very small reflected fields. We will suggest that this result may pave the way for internal antenna control of slot antenna steering.

Assume a fixed wavenumber $k=\frac{\omega}{c}$ where $c$ denotes the speed of light and $\omega$ represents the angular frequency. In this discussion we treat fields that are time-harmonic of the form $e^{-i \omega t}$. In the case of a circular waveguide the time-independent part of the normal modes with frequency $\omega=c k$ is represented by the vector wave functions as follows (see [14], Section 8.7):

$$
\begin{aligned}
& T E_{l n} \text { mode }-\mathbf{E}_{l n}=\nabla \times\left(\mathbf{i} \psi_{l n}\right), \quad \mathbf{B}_{l n}=-\frac{i}{c k} \nabla \times \mathbf{E}_{l n} \\
& T M_{l n} \text { mode }-\mathbf{B}_{l n}=\nabla \times\left(\mathbf{i} \psi_{l n}\right), \quad \mathbf{E}_{l n}=\frac{i c}{k} \nabla \times \mathbf{B}_{l n}
\end{aligned}
$$

where $\mathbf{i}$ denotes the unit vector in the positive $O x$ direction, $i=\sqrt{-1}$,

$$
\psi_{l n}(\mathbf{r})=A_{l n} J_{l}\left(\frac{\chi_{l n}}{R} r\right) \cos (l \varphi) e^{-i \beta_{l n} x}, \text { and } k^{2}=\frac{\chi_{l n}^{2}}{R^{2}}+\beta_{l n}^{2}
$$

with $A_{l n}$ - the amplitude of the $\ln$ mode, $J_{l}$ - the $l$-th Bessel function of the first kind and $\chi_{l n}$ - the $n$-th root of: $J_{l}$ for TM modes and $J_{l}^{\prime}$ for TE modes, respectively. Note that $\psi_{l n}$ is a solution of the Helmholtz equation in $D$ with $\psi_{l n}=0$ on $\partial B_{R}(\mathbf{0})$ and $B_{R}(\mathbf{0})$ is the disk cross section of the wave guide as defined before.

We address Question 1 in two steps:

- Step 1. For every given $T M_{l n}$ or $T E_{l n}$ mode show that boundary data $v_{l n}$ exists, such that $u_{l n}$, the unique radiating solution of the free space exterior Dirichlet problem for the Helmholtz equation with $v_{l n}$ specified on the antenna $\partial D_{a}$, has the property that it almost cancels $\psi_{l n}$ (defined at (2.3)) in the control region $D_{c}$ while itself and its first and second order partial derivatives will have vanishingly small values on the wall of the guide $\partial D$. 
- Step 2. Based on the result of Step 1, we synthesize an antenna $D_{a}$, modeled as a surface tangential field $\mathbf{M}(\mathbf{x})=\mathbf{E} \times \boldsymbol{\nu}$ where $\boldsymbol{\nu}$ denotes the exterior normal to $\partial D_{a}$, such that the radiated electric or magnetic field will be very close to,

$$
\begin{gathered}
-\frac{i c}{k} \sum_{l, n} \nabla \times \nabla \times\left(\mathbf{i} \psi_{l n}\right) \text { for TM, or to } \\
\frac{i}{c k} \sum_{l, n} \nabla \times \nabla \times\left(\mathbf{i} \psi_{l n}\right) \text { for TE respectively, }
\end{gathered}
$$

and thus, as implied by (2.1) and (2.2), we will obtain the desired control properties.

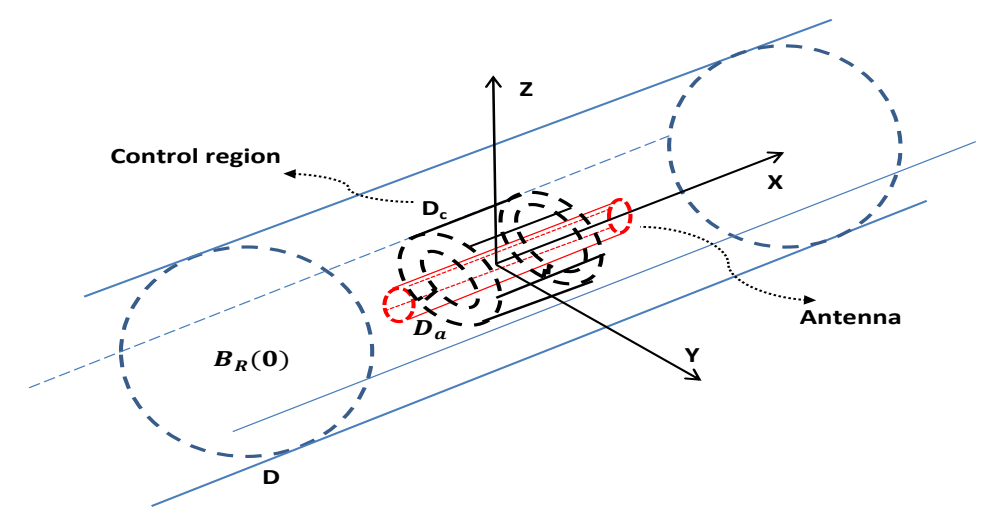

Figure 1: The geometrical setting of the problem where, for simplification, the antenna and control regions appear with straight edges.

Our approach to this problem then starts with a question in electromagnetic theory, namely, can a waveguide mode be influenced by an interior antenna in a controlled manner, moves to a question concerning exterior scalar fields, and then returns to the original electromagnetic control issue. Thus, in the next section we show the existence of boundary data that provides the control of the needed scalar guide mode in the vicinity of the antenna with small field reflected from the waveguide walls.

\subsection{Existence results}

We note that, by using the superposition principle, it is enough to analyze only one normal mode. We will focus on TM modes and show the result for TE modes in Corollary 2.4.

For arbitrarily fixed indexes $p, q$, consider the $\mathrm{TM}_{p q}$ mode of an incoming $\mathrm{TM}$ wave $\left(\mathbf{B}_{i}, \mathbf{E}_{i}\right)$ given by,

$$
\mathbf{B}_{i}=\nabla \times\left(\mathbf{i} \psi_{p q}\right) \text { and } \mathbf{E}_{i}=\frac{i c}{k} \nabla \times \mathbf{B}_{i}
$$

where $\psi_{p q}$ is given at (2.3). Mathematical formulation of the existence issue Step 1 of Question 1 follows: 
Mathematical equivalent of Step 1. Let $0<\varepsilon \ll 1$ and $\psi_{p q}$ described at (2.3).

Find $v_{p q} \in C\left(\partial D_{a}\right)$ such that there exists $u \in C^{2}\left(\mathbb{R}^{3} \backslash \bar{D}_{a}\right) \cap C\left(\mathbb{R}^{3} \backslash D_{a}\right)$ satisfying,

$$
\left\{\begin{array}{l}
\Delta u+k^{2} u=0 \text { in } \mathbb{R}^{3} \backslash \bar{D}_{a}, \\
u=v_{p q} \text { on } \partial D_{a}, \\
\left.\left(\frac{x}{|x|}, \nabla u(x)\right)\right)-i k u(x)=o\left(\frac{1}{|x|}\right), \text { as }|x| \rightarrow \infty, \text { uniformly for all } \frac{x}{|x|}
\end{array}\right.
$$

and

$$
\|u\|_{L^{2}(\partial D)}+\left\|\frac{\partial u}{\partial x_{j}}\right\|_{L^{2}(\partial D)}+\left\|\frac{\partial^{2} u}{\partial x_{i} \partial x_{j}}\right\|_{L^{2}(\partial D)} \leq \varepsilon,\left\|u+\psi_{p q}\right\|_{C^{2}\left(\bar{D}_{c}\right)} \leq \varepsilon .
$$

for all $i, j=\overline{1,3}$.

It is known that, (see [7]), given $v_{b} \in C\left(\partial D_{a}\right)$, problem (2.5) has a unique solution in $C^{2}\left(\mathbb{R}^{3} \backslash \bar{D}_{a}\right) \cap C\left(\mathbb{R}^{3} \backslash D_{a}\right)$. Thus, it remains to be proved that one can find boundary data $v_{p q} \in C\left(\partial D_{a}\right)$ so that the solution of (2.5) satisfies the estimates (2.6) which implies that the local field control has occurred without the generation of significant fields reflected from the waveguide walls.

Let $\xi<<1, L>>1$ fixed and consider a smooth compact domain $D_{L} \subset \mathbb{R}^{3}$ satisfying

$$
(-L, L) \times B_{R-2 \xi}(\mathbf{0}) \Subset D_{L} \Subset(-2 L, 2 L) \times B_{R-\xi}(\mathbf{0})=V_{L}
$$

In what follows we make the assumption that the antenna, the regions of control and the boundary of the waveguide are sufficiently well separated, i.e., there exist $D_{1}$ and $D_{a^{\prime}}$ subdomains of $\mathbb{R}^{3}$ so that

$$
\begin{gathered}
D_{c} \Subset D_{1} \Subset D_{L} \backslash \bar{D}_{a} \Subset V_{L} \backslash \bar{D}_{a} \Subset D \backslash \bar{D}_{a} \\
D_{a^{\prime}} \Subset D_{a}
\end{gathered}
$$

Observe that by construction, from $L>>1$ and the fact that the solution $u \in C^{2}\left(\mathbb{R}^{3} \backslash \bar{D}_{a}\right)$ of (2.5) satisfies the outgoing radiation condition the first inequality in (2.6) is satisfied in $L^{2}(\partial D \backslash\{|x|<L\})$ and (2.6) will be implied if we show,

$$
\begin{aligned}
& \|u\|_{L^{2}(\partial D \cap\{|x|<L\})}+\left\|\frac{\partial u}{\partial x_{j}}\right\|_{L^{2}(\partial D \cap\{|x|<L\})}+\left\|\frac{\partial^{2} u}{\partial x_{i} \partial x_{j}}\right\|_{L^{2}(\partial D \cap\{|x|<L\})} \leq \varepsilon, \\
& \left\|u+\psi_{p q}\right\|_{C^{2}\left(\bar{D}_{c}\right)} \leq \varepsilon .
\end{aligned}
$$

for all $i, j=\overline{1,3}$, where $\{|x|<L\}=\left\{\mathbf{x}=(x, y, z) \in \mathbb{R}^{3},|x|<L\right\}$. We also make the following additional assumptions:

$-k^{2}$ is not a Neumann eigenvalue for the Laplacian in $D_{a^{\prime}}$ or $D_{L}$,

$-k^{2}$ is not a Dirichlet eigenvalue for the Laplacian in $D_{1}$.

Note that, for given $k$, the sets $D_{1}, D_{a^{\prime}}$ and $D_{L}$ can be chosen so that (2.12) and (2.11) are satisfied. As in [44] let us introduce the following space $\Xi$,

$$
\Xi \equiv L^{2}\left(\partial D_{1}\right) \times L^{2}\left(\partial D_{L}\right)
$$


Then $\Xi$ is a Hilbert space with respect to the scalar product given by

$$
(\varphi, \psi)_{\Xi}=\int_{\partial D_{1}} \varphi_{1}(\mathbf{y}) \bar{\psi}_{1}(\mathbf{y}) d s_{\mathbf{y}}+\int_{\partial D_{L}} \varphi_{2}(\mathbf{y}) \bar{\psi}_{2}(\mathbf{y}) d s_{\mathbf{y}}
$$

for all $\varphi \equiv\left(\varphi_{1}, \varphi_{2}\right)$ and $\psi=\left(\psi_{1}, \psi_{2}\right)$ in $\Xi$ where $(\cdot)$ above, denotes the complex conjugate. By using results in [44] we obtain,

Proposition 2.1. Let $k$ satisfying (2.11) and (2.12) and assume $L$ is large enough. Consider the following integral operator, $K: L^{2}\left(\partial D_{a^{\prime}}\right) \rightarrow \Xi$, defined as

$$
K z(\mathbf{x}, \mathbf{z})=\left(K_{1} z(\mathbf{x}), K_{2} z(\mathbf{z})\right)
$$

for any $z \in L^{2}\left(\partial D_{a^{\prime}}\right)$, where

$$
\begin{aligned}
& K_{1} z(\mathbf{x})=\int_{\partial D_{a^{\prime}}} z(\mathbf{y}) \frac{\partial \Phi(\mathbf{x}, \mathbf{y})}{\partial \boldsymbol{\nu}_{\mathbf{y}}} d s_{\mathbf{y}}, \text { for } \mathbf{x} \in \partial D_{1}, \\
& K_{2} z(\mathbf{z})=\int_{\partial D_{a^{\prime}}} z(\mathbf{y}) \frac{\partial \Phi(\mathbf{z}, \mathbf{y})}{\partial \boldsymbol{\nu}_{\mathbf{y}}} d s_{\mathbf{y}}, \text { for } \mathbf{z} \in \partial D_{L},
\end{aligned}
$$

with $\boldsymbol{\nu}_{\mathbf{y}}$ denoting the normal exterior to $\partial D_{a^{\prime}}$ and where $\Phi(\mathbf{x}, \mathbf{y})$ represents the fundamental solution of the relevant Helmholtz operator, i.e.,

$$
\Phi(\mathbf{x}, \mathbf{y})=\frac{e^{i k|\mathbf{x}-\mathbf{y}|}}{4 \pi|\mathbf{x}-\mathbf{y}|}
$$

The operator $K$ is compact and has a dense range. Moreover, for $\alpha_{n} \rightarrow 0$ (i.e., Tikhonov regularizers) the functions $v_{n} \in C\left(\partial D_{a}\right)$ defined by

$$
v_{n}=\left(\alpha_{n} I+K^{*} K\right)^{-1} K^{*} v \text {, with } v=\left(-\psi_{p q}, 0\right),
$$

have the property that, for any $\delta<<1$, there exists an $N_{\varepsilon} \in \mathbb{N}$ such that for any $n>N_{\varepsilon}$ the functions $u_{n}$ given by,

$$
u_{n}=\int_{\partial D_{a^{\prime}}} v_{n}(\mathbf{y}) \frac{\partial \Phi(\mathbf{x}, \mathbf{y})}{\partial \nu_{\mathbf{y}}} d s_{\mathbf{y}}
$$

is such that $u_{n} \in C^{2}\left(\mathbb{R}^{3} \backslash \bar{D}_{a^{\prime}}\right)$ and satisfies,

$$
\left\{\begin{array}{l}
\Delta u_{n}+k^{2} u_{n}=0, \text { in } \mathbb{R}^{3} \backslash \bar{D}_{a} \\
\left\|u_{n}\right\|_{C^{2}\left(\mathbb{R}^{3} \backslash V_{L}\right)} \leq \varepsilon \\
\left\|u_{n}+\psi_{p q}\right\|_{C^{2}\left(D_{c}\right)} \leq \varepsilon
\end{array}\right.
$$

where $V_{L}$, with $D_{L} \Subset V_{L} \Subset D$, was introduced at (2.7). 
Proof. The proof follows directly from Theorem 2.1, Corollary 2.2, and Remark 2.3 in [44] for the case of $n=1$ and Geometry II. (see (2.2) page 555 in [44]).

Indeed, consider the linear compact integral operator $K$ defined at $(2.15),(2.16)$. It is implied by [44] that, with the hypothesis of the Proposition, this operator is injective and has a dense range. Following [44] we conclude that this implies that, in theory, there exists a sequence of solutions of (2.20). In fact, as showed in [44], the problem can be formulated as a Fredholm equation of the first kind for $\mathrm{K}$ in $\Xi$ whose Tikhonov regularization minimal energy solution $([15,4])$ is $u_{n}$ defined at (2.19) and (2.18). This, together with classical regularity results shows that the unique radiating solution of (2.20) (i.e., satisfying the Sommerfeld radiation condition) with boundary data given by $\int_{\partial D_{a^{\prime}}} v_{n}(\mathbf{y}) \frac{\partial \Phi(\mathbf{x}, \mathbf{y})}{\partial \nu_{\mathbf{y}}} d s_{\mathbf{y}}$ on $\partial D_{a}$ (with $v_{n}$ defined at (2.18)) is $u_{n}$.

Then, the conclusion follows by observing that from the smoothness of $u n$, the fact that $D_{L} \Subset V_{L} \Subset D$ and (2.20) we obtain (2.10) which in turn implies (2.6).

The following result shows the existence of a minimal energy solution for (2.20) and follows immediately from Proposition 2.1 by using similar ideas as in [44] or [45].

Corollary 2.1. Let $v=\left(-\psi_{p q}, 0\right) \in \Xi$ as in Proposition 2.1 and consider $0<\epsilon<<1$ arbitrary. Then there exists $u_{0} \in C\left(\partial D_{a^{\prime}}\right)$ such that,

$$
\left\|u_{0}\right\|_{L^{2}\left(\partial D_{a^{\prime}}\right)}=\min _{\|K u-v\|_{\Xi \leq \epsilon}}\|u\|_{L^{2}\left(\partial D_{a^{\prime}}\right)}
$$

Remark 2.2. Note that by construction, since $D_{a^{\prime}} \Subset D_{a}$, it can be observed that $u_{n}$ will be infinitely differentiable on $\partial D_{a}$.

The next Remark finalizes the proof of the Step 1 of Question 1.

Remark 2.3. Proposition 2.1 (definition given at (2.19)) and uniqueness for the scalar exterior problem imply that $u_{n}$ is the unique solution of (2.5) for $v_{p q}=u_{n}$ on $\partial D_{a}$. Choosing $n>N_{\varepsilon}$, where $N_{\varepsilon}$ is introduced in Proposition 2.1, it follows from from (2.7) and (2.20) that $u_{n}$ will also satisfy estimates (2.6).

We thus have completed Step1 of our study of whether an antenna can be placed within a circular cylindrical waveguide to null a waveguide mode near the antenna without generating significant reflection from the inner wall of the waveguide. We have thus far shown that the scalar potential associated with the waveguide mode can be approximated by a solution of the Helmholtz equation with an infinitesimally small residual scalar field at the waveguide wall. Now we transition to the electromagnetic field and present the argument for the proof for Step2 of Question 1.

Let $\left\{u_{n}\right\}_{n}$ be the sequence introduced at (2.19). For arbitrary fixed $n>N_{\varepsilon}$, consider 
the following exterior Maxwell boundary- value problem (see Section 4.3. in [7]),

$$
\begin{cases}\nabla \times \tilde{\mathbf{E}}-i k \tilde{\mathbf{H}}=0, \nabla \times \tilde{\mathbf{H}}+i k \tilde{\mathbf{E}}=0 & \text { in } \mathbb{R}^{3} \backslash \bar{D}_{a} \\ \tilde{\mathbf{E}} \times \boldsymbol{\nu}=\frac{i \sqrt{\epsilon_{0}} c}{k}\left(\nabla \times \nabla \times\left(\mathbf{i} u_{n}\right)\right) \times \boldsymbol{\nu}, & \text { on } \partial D_{a} \\ {\left[\tilde{\mathbf{H}}, \frac{\mathbf{x}}{|\mathbf{x}|}\right]-\tilde{\mathbf{E}}=o\left(\frac{1}{|\mathbf{x}|}\right),} & \text { as }|\mathbf{x}| \rightarrow \infty \\ {\left[\tilde{\mathbf{E}}, \frac{\mathbf{x}}{|\mathbf{x}|}\right]+\tilde{\mathbf{H}}=o\left(\frac{1}{|\mathbf{x}|}\right),} & \text { as }|\mathbf{x}| \rightarrow \infty\end{cases}
$$

where $\boldsymbol{\nu}$ is the unit exterior normal to $D_{a}, \tilde{\mathbf{E}}=\sqrt{\epsilon_{0}} \mathbf{E}$ and $\tilde{\mathbf{H}}=\sqrt{\mu_{0}} \mathbf{H}$, with $\mathbf{E}$ and $\mathbf{H}=\mu_{0}^{-1} \mathbf{B}$ representing the time independent part of the physical electric and magnetic fields generated by $\mathbf{E} \times \boldsymbol{\nu}=\frac{i c}{k}\left(\nabla \times \nabla \times\left(\mathbf{i} u_{n}\right)\right) \times \boldsymbol{\nu}$, on $\partial D_{a}$ and with $\epsilon_{0}, \mu_{0}$ denoting the electric permitivity and respectively the magnetic permeability of air. Is is shown in [7] (see Section 4.3) that problem (2.21) has a unique solution $\tilde{\mathbf{E}}, \tilde{\mathbf{H}} \in C^{1}\left(\mathbb{R}^{3} \backslash \bar{D}_{a}\right) \cap C\left(\mathbb{R}^{3} \backslash D_{a}\right)$. Let $\tilde{\mathbf{E}}_{i}=\sqrt{\epsilon_{0}} \mathbf{E}_{i}$ and $\tilde{\mathbf{H}}_{i}=\sqrt{\mu_{0}} \mathbf{H}_{i}$ with $\mathbf{E}_{i}$ and $\mathbf{H}_{i}=\mu_{0}^{-1} \mathbf{B}_{i}$ representing the incident electromagnetic field introduced in (2.4). To complete Step2 of Question 1 it remains to be proved that the unique solution of (2.21) satisfies

$$
\begin{aligned}
& \left\|\tilde{\mathbf{E}}+\tilde{\mathbf{E}}_{i}\right\|_{C\left(\bar{D}_{c}\right)} \leq C \epsilon, \quad\left\|\tilde{\mathbf{H}}+\tilde{\mathbf{H}}_{i}\right\|_{C\left(\bar{D}_{c}\right)} \leq C \epsilon \\
& \|\tilde{\mathbf{E}}\|_{C(\partial D)} \leq C \epsilon, \quad\|\tilde{\mathbf{H}}\|_{C(\partial D)} \leq C \epsilon
\end{aligned}
$$

for some constant $C$. Indeed, from proposition 2.1, Remark 2.3 and the above characterization for the solutions of (2.21) we obtain:

Theorem 2.1. Let $N_{\varepsilon}$ be as in Proposition 2.1. For arbitrary fixed $n>N_{\varepsilon}$, the tangential field given by $\mathbf{E} \times \boldsymbol{\nu}=\frac{i c}{k}\left(\nabla \times \nabla \times\left(\mathbf{i} u_{n}\right)\right) \times \boldsymbol{\nu}$ on $\partial D_{a}$, where the sequence $\left\{u_{n}\right\}_{n}$ is defined at (2.19) (with $\psi_{p q}$ as in (2.3) for TM), generates a field, $(\mathbf{B}, \mathbf{E})$ in the exterior of the antenna $D_{a}$, with $\mathbf{E}=\frac{i c}{k}\left(\nabla \times \nabla \times\left(\mathbf{i} u_{n}\right)\right)$ and $\mathbf{B}=\nabla \times\left(\mathbf{i} u_{n}\right)$. Moreover we have

$$
\begin{aligned}
& \left\|\mathbf{E}+\mathbf{E}_{i}\right\|_{C\left(\bar{D}_{c}\right)} \leq C \epsilon, \quad\left\|\mathbf{B}+\mathbf{B}_{i}\right\|_{C\left(\bar{D}_{c}\right)} \leq C \epsilon, \\
& \|\mathbf{E}\|_{C(\partial D)} \leq C \epsilon, \quad\|\mathbf{B}\|_{C(\partial D)} \leq C \epsilon,
\end{aligned}
$$

where $C$ is a constant depending only on $c, k$ and $\left(\mathbf{E}_{i}, \mathbf{B}_{i}\right)$ are defined at (2.4) with with $\psi_{p q}$ as in at (2.3) for TM.

Proof. From (2.4) we have that

$$
\mathbf{B}_{i}=\nabla \times\left(\mathbf{i} \psi_{p q}\right), \tilde{\mathbf{H}}_{i}=\frac{1}{\sqrt{\mu_{0}}} \mathbf{B}_{i}, \mathbf{E}_{i}=\frac{i c \mu_{0}}{k} \nabla \times \mathbf{H}_{i} \text { and } \tilde{\mathbf{E}}_{i}=\sqrt{\epsilon_{0}} \mathbf{E}_{i} .
$$

By using the Sommerfeld radiation condition satisfied by $u_{n}$ and careful asymptotic estimates for $|\mathbf{x}| \rightarrow \infty$ one can show that $\tilde{\mathbf{E}}=\frac{i \sqrt{\epsilon_{0}} c}{k}\left(\nabla \times \nabla \times\left(\mathbf{i} u_{n}\right)\right)$ and $\tilde{\mathbf{H}}=c \sqrt{\epsilon_{0}} \nabla \times\left(\mathbf{i} u_{n}\right)$ satisfy $(2.21)$ and by uniqueness (see Section 4.3. in [7]) it follows that they are the unique solutions of $(2.21)$. 
On the other hand we showed above in Proposition 2.1 and Remark 2.3 that $u_{n}$ are a class of solutions for Step 1 of Question 1 and thus satisfy equation (2.5) and estimates (2.6). This will imply the fact that the unique solutions of $(2.21),(\tilde{\mathbf{E}}, \tilde{\mathbf{H}})$, also satisfy estimate (2.22). The conclusion of the theorem follows by recalling that $\tilde{\mathbf{E}}=\sqrt{\epsilon_{0}} \mathbf{E}$ and $\tilde{\mathbf{H}}=\sqrt{\mu_{0}} \mathbf{H}$, $\tilde{\mathbf{E}}_{i}=\sqrt{\epsilon_{0}} \mathbf{E}_{i}$ and $\tilde{\mathbf{H}}_{i}=\sqrt{\mu_{0}} \mathbf{H}_{i}, \mathbf{H}_{i}=\mu_{0}^{-1} \mathbf{B}_{i}$ and $\tilde{\mathbf{H}}_{i}=\mu_{0}^{-1} \tilde{\mathbf{B}}_{i}$.

As we mentioned in the beginning of the section, by using the above ideas adapted to the TE context we obtain a similar result for TE modes. Indeed we have,

Corollary 2.4. For arbitrary fixed $n>N_{\varepsilon}$ (where $N_{\varepsilon}$ is introduced above), the tangential field given by $\mathbf{E} \times \boldsymbol{\nu}=\left(\nabla \times\left(\mathbf{i} u_{n}\right)\right) \times \boldsymbol{\nu}$ on $\partial D_{a}$, where the sequence $\left\{u_{n}\right\}_{n}$ is defined at (2.19) (with $\psi_{p q}$ as in (2.3) for TE), generates a field, $(\mathbf{B}, \mathbf{E})$ in the exterior of the antenna $D_{a}$, with $\mathbf{E}=\nabla \times\left(\mathbf{i} u_{n}\right)$ and $\mathbf{B}=-\frac{i}{c k} \nabla \times \nabla \times\left(\mathbf{i} u_{n}\right)$. Moreover we have

$$
\begin{aligned}
& \left\|\mathbf{E}+\mathbf{E}_{i}\right\|_{C\left(\bar{D}_{c}\right)} \leq C \epsilon, \quad\left\|\mathbf{B}+\mathbf{B}_{i}\right\|_{C\left(\bar{D}_{c}\right)} \leq C \epsilon \\
& \|\mathbf{E}\|_{C(\partial D)} \leq C \epsilon, \quad\|\mathbf{B}\|_{C(\partial D)} \leq C \epsilon
\end{aligned}
$$

where again $C$ is a constant depending only on $c, k$ and now, in the TE case, $\left(\mathbf{E}_{i}, \mathbf{B}_{i}\right)$ are defined by

$$
\mathbf{E}_{i}=\nabla \times\left(\mathbf{i} \psi_{p q}\right), \quad \mathbf{B}_{i}=-\frac{i}{c k} \nabla \times \mathbf{E}_{i}
$$

with $\psi_{p q}$ as in (2.3) for TE..

\section{Feasibility discussion}

Remark 3.1. The discussion in this section is not a numerical analysis of the ill-posed problem proposed in Question 1. This is an interesting investigation and we will consider it in a forthcoming paper. Instead we develop a feasibility discussion for the theoretical results obtained in Section 2 to show that:

1. The surface currents predicted in Theorem 2.1 (which in general is not divergence free) can in principle be approximated with a layer of physical dipoles (each of particular orientation, current amplitude and phase), Lemma 3.1.

2. In the case of the radially symmetric TM modes, we show how in principle a simple small cylindrical array of angularly equi-distributed dipoles, each parallel with the waveguide axis, can in principle null the longitudinal component of the electrical field in a prescribed volume near the array support.

3. Such control results could in principle be used to enhance the versatility of slotted waveguide arrays.

We next ask whether the electric and magnetic fields generated by the scalar function $u_{n}$ as described in Theorem 2.1 can be realized using realistic current carrying structures. 
We will focus on the TM regime at fixed wavenumber $\lambda$ satisfying the condition (2.11) and (2.12).

We approach the feasibility issue by first considering whether the solution proposed in Theorem 2.1, i.e., tangential vectors given by $\mathbf{E} \times \boldsymbol{\nu}=\frac{i c}{k}\left(\nabla \times \nabla \times\left(\mathbf{i} u_{n}\right)\right) \times \boldsymbol{\nu}$, on $\partial D_{a}$, can be understood as a continuum of physical currents. Then we explore approximating these currents by a local discrete set of antennas.

In what follows we will study the principal TM mode, i.e., $\mathrm{TM}_{01}$ mode. It is a direct matter to determine that the tangential field given by $\mathbf{E} \times \boldsymbol{\nu}=\frac{i c}{k}\left(\nabla \times \nabla \times\left(\mathbf{i} u_{n}\right)\right) \times \boldsymbol{\nu}$, on $\partial D_{a}$ is not a physical surface magnetic current since their surfacic divergence does not vanish in general (see [40], page 158). Nevertheless they can be physically approximated as shown in [7] (Theorem 2.26, Theorem 4.19). Thus we conclude,

Lemma 3.1. Assume $k$ is not an interior Maxwell eigenvalue for $D_{a}$ (as defined in [7]). Then any smooth enough tangential field on $\partial D_{a}$ can be approximated with a layer of dipoles.

Proof. We will consider only the case of magnetic dipoles the other case following similarly. The proof of Lemma 3.1 follows from standard jump conditions for vector potentials (see [7]). Indeed, recall that the electromagnetic field due to a layer of magnetic dipoles distributed on $\partial D_{a}$ with tangential density $\mathbf{b} \in C^{0, \alpha}\left(\partial D_{a}\right)$ for some $0<\alpha<1$ is given by,

$$
\tilde{\mathbf{E}}(\mathbf{x})=\nabla \times \int_{\partial D_{a}} \mathbf{b}(\mathbf{y}) \Phi(\mathbf{x}, \mathbf{y}) d s_{\mathbf{y}}, \quad \tilde{\mathbf{H}}(\mathbf{x})=\frac{1}{i k} \nabla \times \tilde{\mathbf{E}}(\mathbf{x})
$$

where as before $\tilde{\mathbf{E}}=\sqrt{\epsilon_{0}} \mathbf{E}$ and $\tilde{\mathbf{H}}=\sqrt{\mu_{0}} \mathbf{H}$. Following [7] we have that for any tangential field $\mathbf{d}: \partial D_{a} \rightarrow \mathbb{C}^{3}$ with $(\mathbf{d}, \boldsymbol{\nu})=0$ (where $\boldsymbol{\nu}$ denotes the exterior normal to $\left.\partial D_{a}\right), \mathbf{d} \in C^{0, \alpha}\left(\partial D_{a}\right.$ ) and Div $\mathbf{d} \in C^{0, \alpha}\left(\partial D_{a}\right)$ (where Div denotes the surfacic divergence as defined in [7]), there exists $\mathbf{b} \in C^{0, \alpha}\left(\partial D_{a}\right)$ such that

$$
\lim _{h \rightarrow 0+}\left(\boldsymbol{\nu}(\mathbf{x}) \times\left(\nabla \times \int_{\partial D_{a}} \mathbf{b}(\mathbf{y}) \Phi(\mathbf{x}+h \boldsymbol{\nu}(\mathbf{x}), \mathbf{y}) d s_{\mathbf{y}}\right)\right)=\mathbf{d}(\mathbf{x}), \text { uniformly on } \partial D_{a}
$$

where $h \rightarrow 0+$ denote $h \rightarrow 0$ with $h>0$. This follows from Theorem 2.26, Theorem 4.19 and Theorem 4.23 in [7]. Observing that in our case $\mathbf{d}=\frac{i c}{k}\left(\nabla \times \nabla \times\left(\mathbf{i} u_{n}\right)\right) \times \boldsymbol{\nu}$ satisfies the above smoothness assumption since $u_{n}$ are smooth on $\partial D_{a}$ (recall that $D_{a^{\prime}} \subset \subset D_{a}$ ), from (3.28) and (3.27) we obtain the statement of the Lemma 3.1.

The relation (3.28) means that the electromagnetic field generated by the surface current introduced in Theorem 2.1, can be approximated by the field corresponding to a layer of dipoles ( having divergence free surface current) by small alteration of the boundary data. Thus, from the stability results of the exterior Maxwell problem with respect to small perturbations in the boundary data, we are assured that the physically meaningful dipole layer current on the antenna surface will produce the desired control effects. Now we are ready to face the issue of whether this continuum current can be adequately replaced by a discrete array of current carrying elements.

The above discussion provides impetus for an attempt to locally null a transverse magnetic mode within a cylindrical waveguide using a small array of dipoles. In the following 
discussion we will show how, in principle, such an array could be built to obtain the desired effect in the case of radially symetric $T M$ modes. To do so we will focus on the $T M_{01}$ mode and in Remark 3.3 we will note how our discussion extends to all $T M_{0 n}$ modes defined at (2.3). In the following we discuss the array structure and the mode it is designed to control.

We, will consider a small array of electric dipoles. The array is 5 centimeters long and 12 centimeters in diameter centered on the long axis, in the center, of an infinitely long perfectly conducting cylinder of radius 50 centimeters. We apply this array to the $T M_{01}$ mode of the cylinder. This mode is 1 volt per meter at the center of the waveguide and zero at the boundary of the cylindrical wall.

Working at 2.5 times the waveguide cutoff frequency $(573.7187 \mathrm{Mhz})$, the field peak voltage around the interior small array due to the $T M_{01}$ mode in the waveguide (with the antenna unpowered) is provided in Figure 2. The numerical values in this figure were computed according to the $T M_{l n}$ mode equation

$$
E_{x}=-i B_{l n} \frac{\beta_{\rho, l n}^{2}}{\omega \mu \epsilon} J_{l}\left(\rho \beta_{\rho, l n}\right)[C \cos (l \varphi)] e^{-i z \beta_{z, l n}}
$$

with $\beta_{\rho, l n}=\frac{\chi_{l n}}{a}$ and $\beta_{z, l n}=\sqrt{\left(\frac{w}{c}\right)^{2}-\beta_{\rho, m n}^{2}}$ and where $\chi_{l n}$ was defined at (2.3).

In these equations we have slightly adapted the symbol use of in [5] (page 478). The radius of the cylinder is $a$, and the $n$-th zero of $J_{l}$ is $\chi_{l n}$. The coefficients $B_{l n}$ and $C$ above were chosen so that the field has unity amplitude at the center of the cylinder.

Referring to Figure 2, recall that the interior array is 12 centimeters in diameter or six centimeters in radius. Peak field amplitude is constant with longitudinal distance as expected. The field phase is constant with radius as expected, but varies with $x$ distance along the cylindrical waveguide as shown in Figure 3.

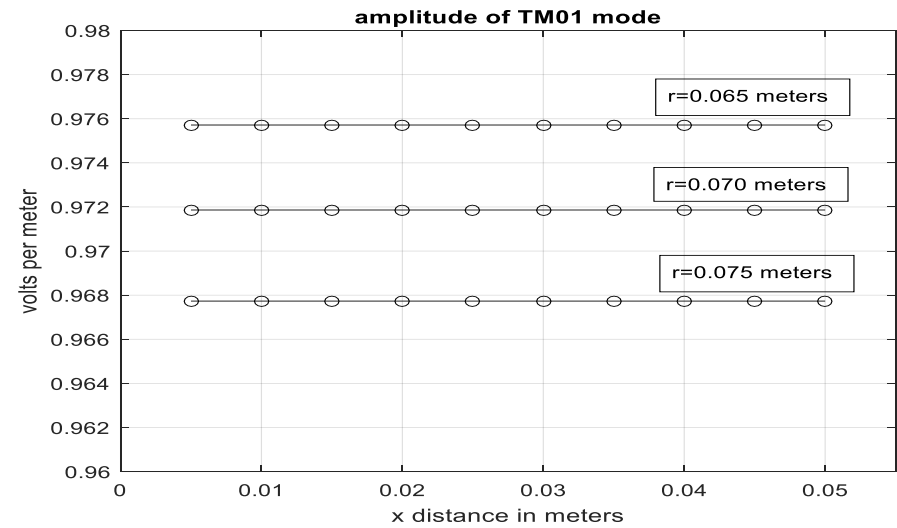

Figure 2: The TM $M_{01}$ field amplitude along a length of the cylindrical waveguide to the left and right of its center point $x=0.025$.

Figures 2 and 3 describe the field that the emplaced array structure will have to null to provide evidence to support the above theory development. Thus, especially from Figure 3, we see that the array, if successful, will have to cope with a time and space varying signal. 


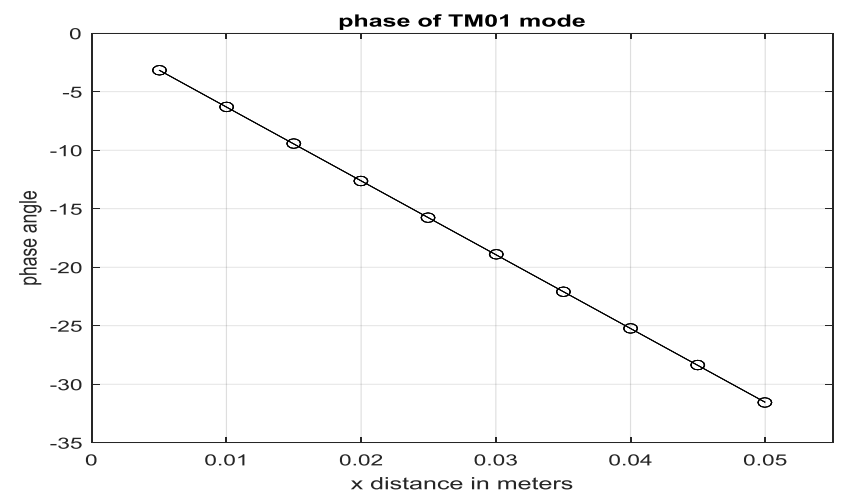

Figure 3: $T M_{01}$ field phase along a length of the cylindrical waveguide to the left and right of its center point. The phase of this mode is constant with radial distance in the waveguide.

Can we put an antenna structure within the cylinder which can, at least on a thin local surface, quench or null (destructively interfere) with the $T M_{01}$ mode pictured in Figure 2 and Figure 3?

We have started with a single infinitesimal current element placed eccentrically in the infinite cylindrical waveguide. The equation we have used to compute the field of this eccentrically placed dipole whose axis is parallel to the long axis of the cylinder, is: [41], (see also $[49,53])$ :

$$
\begin{aligned}
\mathbf{E}_{x}\left(\mathbf{r}, \mathbf{r}_{0}\right) & =\alpha \sum_{j=1}^{\infty} \frac{1}{J_{0}^{\prime}\left(\chi_{0 j}\right)^{2}} \frac{\chi_{0 j}^{2}}{a \gamma_{0 j}} J_{0}\left(\frac{r}{a} \chi_{0 j}\right) e^{i \gamma_{0 j}\left|x-x_{0}\right|} \\
& +\alpha \sum_{n=1}^{\infty} \sum_{j=1}^{\infty} \frac{1}{J_{n}^{\prime}\left(\chi_{n j}\right)^{2}} \frac{\chi_{n j}^{2}}{a \gamma_{n j}} J_{n}\left(\frac{r_{0}}{a} \chi_{n j}\right) J_{n}\left(\frac{r}{a} \chi_{n j}\right) \cos \left[n\left(\varphi-\varphi_{0}\right)\right] e^{i \gamma_{n j}\left|x-x_{0}\right|}
\end{aligned}
$$

This equation is an adaptation of one derived in [41] where the formula is for a dipole within a circular cylindrical waveguide with perfectly conductive walls wherein the dipole has a fully arbitrary orientation within the waveguide. We have simplified the equation to the case of a dipole whose orientation is parallel to the long axis of the waveguide.

In this equation $\mathbf{r}=(x, y, z)=(x, r, \varphi), \mathbf{r}_{0}=\left(x_{0}, y_{0}, z_{0}\right)=\left(x_{0}, r_{0}, \varphi_{0}\right) ; \chi_{n j}$ is the $j$ th root of the $n$-th order Bessel function $J_{n} ; a$ is the diameter of the circular cylindrical waveguide; and $\gamma_{n j}=\sqrt{\left(\frac{\omega}{c}\right)^{2}-\left(\frac{\chi_{n j}}{a}\right)^{2}}$. The applicability of this equation is for $r>r_{0}$ (where $r_{0}$ is the radial distance of the dipole from the center of the cylinder), and $x>x_{0}$ or $x<x_{0}$ (where $x_{0}$ is the $\mathrm{x}$-coordinate of the dipole).

In [41], the author derived this result starting with Debye potentials and ensured that the radiated and reflected fields were such that they summed to exactly zero on the wall of the waveguide. Then, having derived an infinite integral with singular integrand, the residues were located and thus the needed solution was obtained.

In [49], a similar series is derived using residue calculus addressing what seem to be identical integral equations. The series obtained in [49] are such that, in our hands, convergence was not achieved despite the computation of 500 roots for each Bessel function up to order 500. We have also benefited from an earlier reference [53].

Thus we have used (3.30) and performed checks using 4NEC2 (open source MoM electromagnetic software), as will be discussed further. The series in (3.30) does converge but 
as one computes closer to the radiating element, more terms are needed in the mode and root expansion.

Figure 4 below shows our computed field around the infinitesimal array element placed at at $(x, y, z)=(0,0,0.06)$. The dipole element is parallel to the cylindrical waveguide central axis. In this figure we show computations of the dipole radiation pattern in the planes perpendicular to the cylinder axis at $x=0.005, x=0.01$ and $x=0.015$ meters. The pattern in Figure 4 is computed and drawn with origin at $(0,0,0)$ using a radial distance of $r=0.07$ from the cylindrical waveguide axis. We have computed and shown the pattern centered on the axis of the cylindrical waveguide since this is the pattern that is used in the subsequent development of sub-array rings and the complete array to be described. It is interesting to see the pattern shaping occurring so quickly as a function of distance from the source.

As seen in the Figure 5 below, the pattern becomes stable for larger values of $x,(x=$ $0.035, x=0.04$ and $x=0.045)$.

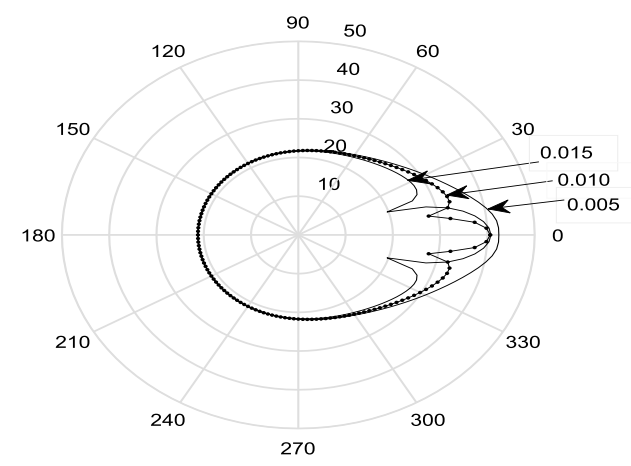

Figure 4: Computations of the dipole radiation pattern in the planes perpendicular to the cylinder axis at $x=0.005, x=0.01$ and $x=0.015$ meters. The pattern is centered on the axis and represents the field amplitude in $d B$ at the radial distance of $r=0.07$ from the cylindrical waveguide axis.

As our next step, we combined eight dipoles in a single plane with placement at 0,45 , $90,135,180,225,270$, and 315 degrees with each dipole at 0.06 off the central waveguide axis (that is, $r_{0}=0.06$ ): all dipoles are oriented parallel to the central axis of the waveguide and are in the plane defined by $x=0$. The resulting pattern for this ring of eight dipoles (a sub-array) is shown below in Figure 6; in this figure black refers to the pattern at $r=0.07$ and $x=0.005$, magenta refers to $r=0.07$ and $x=0.01$, and blue refers to $r=0.07$ and $x=0.015$.

We have computed the fields in planes orthogonal to the waveguide axis and further from the plane of the ring array. The radiation pattern seems to stabilize. This is seen in Figure 7 below where black refers to the pattern at $r=0.07$ and $x=0.035$, magenta refers to $r=0.07$ and $x=0.04$, and blue refers to $r=0.07$ and $x=0.045$. As we have studied the computation we perceive that the engendered field is a combination of evanescent fields that are not propagating and waveguide modes that are propagating. 


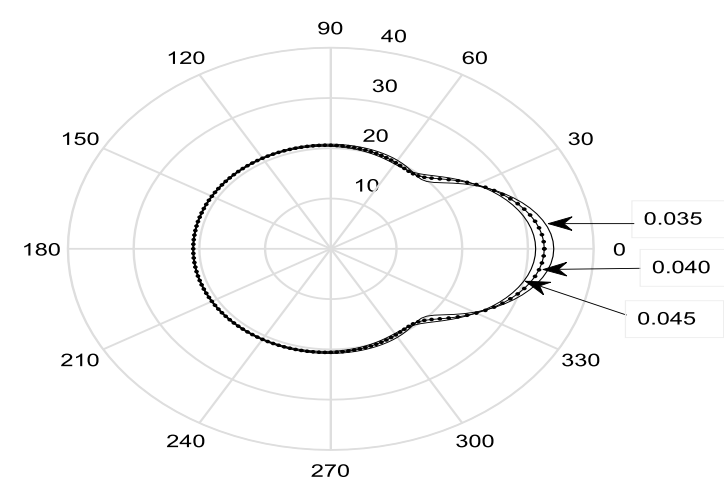

Figure 5: Computations of the dipole radiation pattern in the planes perpendicular to the cylinder axis at $x=0.035, x=0.04$ and $x=0.045$ meters. The pattern is centered on the axis and represents the field amplitude in $d B$ at the radial distance of $r=0.07$ from the waveguide axis.

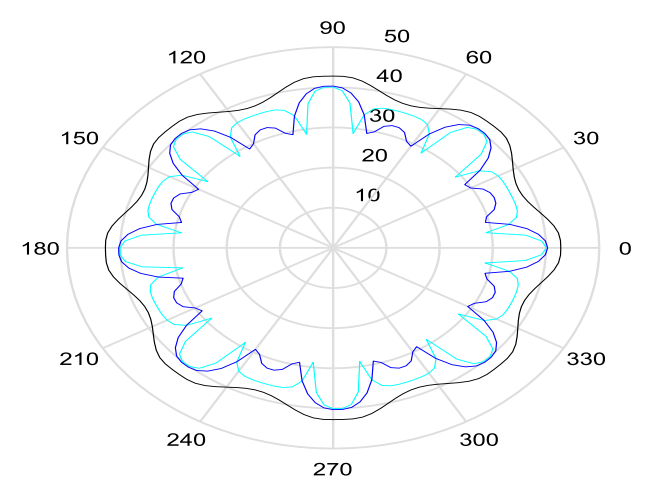

Figure 6: Radiation pattern of a ring of dipoles. Eight dipoles, oriented parallel to the long axis of the cylinder, comprise the ring, evenly spaced at 45 degrees. The dipoles are in the plane defined by $x=0$ and are at a radial distance of 0.060 meters from the waveguide axis. The pattern shown is of the electric field expressed in $d B$ at $r=0.07$ meters or on a cylindrical surface whose radius is one centimeter greater than the radius of the dipole ring. The values shown are for $r=0.07$ and $x=0.005$ (black), to $r=0.07$ and $x=0.01$ (magenta), and $r=0.07$ and $x=0.015$ (blue).

\subsection{Computation of the array currents to null the $T M_{01}$ mode}

Having computationally put together a ring of small (infinitesimal) dipoles we are now in a position to compute the effect of multiple rings combined. Recall that the field of small dipoles are comparable when the length of the dipole is less than approximately $2 \%$ of a wavelength. We have computed the field of three rings (subarrays) together with the rings spaced 0.025 meters (2.5 centimeters) apart using superposition concepts as were used in the formation of the individual subarrays.

Since each array is driven by the same current to each element in each array, we now have three variables which we can use to adjust the fields of each ring in the array to provide a desired local field value. 


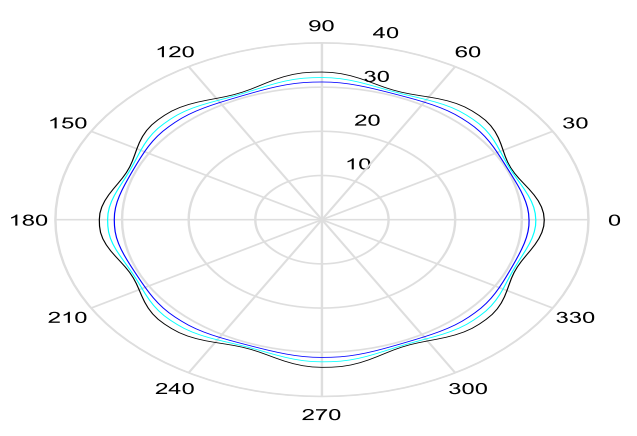

Figure 7: Radiation pattern of a ring of eight dipoles, each dipole oriented parallel to the long axis of the cylinder, evenly spaced at 45 degrees. The dipoles are in the plane defined by $x=0$ and are at a radial distance of 0.060 meters from the waveguide axis. The pattern shown is of the electric field expressed in $d B$ at $r=0.070$ meters or in an cylindrical surface whose radius is one centimeter greater than the radius of the dipole ring. The patterns shown are for $r=0.070$ and $x=0.035$ (black), to $r=0.07$ and $x=0.04$ (magenta), and $r=0.07$ and $x=0.045$ (blue).

The three ring array spans a longitudinal distance of 0.050 meters. We choose to control the field between ring 1 (located at $x=0$ meters) and ring 2 (at $x=0.025$ meters). We choose to work between 19 and 21 degrees as seen on the polar graphs above and at radii between 0.068 and 0.070 . We sampled the waveguide field at thirty six points in the selected spatial volume using equation (3.30), and, using linear regression analysis, we determined the ring currents that provided ring fields that best matched the fields of the $T M_{01}$ mode of the wave guide in that volume [39].

The linear regression analysis was performed using the longitudinal component of $T M_{01}$ field described at (3.29) as the data to be fitted using the 24 element array. At each of thirty six points we computed the complex valued $T M_{01}$ field, for which we use the symbol $E_{z}\left(x_{i}, y_{i}, z_{i}\right)$. Using superposition we defined a regression model such that each $T M_{01}$ field point is approximated as the weighted sum of the fields from each of the three array rings or subarrays of eight dipoles. That is,

$$
E_{x}\left(x_{i}, y_{i}, z_{i}\right) \approx \sum_{j=1}^{3} R\left(x_{i}, y_{i}, z_{i}, j\right) w_{j}
$$

In this relationship, $j$ is the index for the sub-array number. This relationship simply says that the $T M_{01}$ field is being approximated by the sum of the three fields from the three sub-arrays of the total array. Since the sub-array fields, $R\left(x_{i}, y_{i}, z_{i}, j\right)$, have been computed for an input current of one ampere to each dipole element of the array, the weights $w_{j}$ that we compute using this relationship by minimizing the sum of the squared errors, are the best approximating currents in the least squares sense.

Equation (3.31) can be cast in vector/matrix form. We define $\mathbf{E}$ as a column vector of computed array longitudinal field values $E_{x}\left(x_{i}, y_{i}, z_{i}\right)$ in a set of $N$ prescribed points $\left(x_{i}, y_{i}, z_{i}\right), i=\{1, \ldots, N\}$. A matrix $R$ is formed with $R_{i j}$ being given by the field generated 
at the point $\left(x_{i}, y_{i}, z_{i}\right)$ by the $j-t h$ ring at one ampere and we define $\mathbf{W}=\left(W_{1}, W_{2}, W_{3}\right)^{T}$ as the weight vector such that

$$
\mathbf{E}(i)=R_{i j} W_{j}
$$

Then we computed the least square solution for the above system through the associated normal equation,

$$
\mathbf{W}=\left(R^{T} R\right)^{-1} R^{T} \mathbf{E}
$$

\begin{tabular}{|c|c|c|c|c|c|}
\hline angle & Radial position & x-coordinate & Data & I & Model \\
\hline \multirow{12}{*}{19 degrees } & \multirow{4}{*}{0.068} & 0.005 & $0.9720+0.0536 i$ & / & $1.0319+0.0457 i$ \\
\hline & & 0.010 & $0.9675+0.1070 i$ & / & $0.9848+0.0820 i$ \\
\hline & & 0.015 & $0.9602+0.1601 i$ & / & $0.9945+0.1396 i$ \\
\hline & & 0.020 & $0.9499+0.2126 i$ & / & $1.0391+0.2097 i$ \\
\hline & \multirow{4}{*}{0.069} & 0.005 & $0.9711+0.0535 i$ & / & $1.0166+0.0523 i$ \\
\hline & & 0.010 & $0.9668+0.1069 i$ & / & $0.9834+0.0873 i$ \\
\hline & & 0.015 & $0.9594+0.1599 i$ & / & $0.9883+0.1437 i$ \\
\hline & & 0.020 & $0.9492+0.2125 i$ & / & $1.0078+0.2130 i$ \\
\hline & \multirow{4}{*}{0.070} & 0.005 & $0.9704+0.0535 i$ & / & $1.0065+0.0566 i$ \\
\hline & & 0.010 & $0.9660+0.1068 i$ & / & $0.9819+0.0908 i$ \\
\hline & & 0.015 & $0.9586+0.1598 i$ & / & $0.9837+0.1464 i$ \\
\hline & & 0.020 & $0.9484+0.2123 i$ & / & $0.9868+0.2153 i$ \\
\hline \multirow{12}{*}{20 degrees } & \multirow{4}{*}{0.068} & 0.005 & $0.9720+0.0536 i$ & / & $0.9944+0.0571 i$ \\
\hline & & 0.010 & $0.9675+0.1070 i$ & / & $0.9547+0.0923 i$ \\
\hline & & 0.015 & $0.9602+0.1601 i$ & / & $0.9581+0.1484 i$ \\
\hline & & 0.020 & $0.9499+0.2126 i$ & / & $0.9823+0.2173 i$ \\
\hline & \multirow{4}{*}{0.069} & 0.005 & $0.9712+0.0535 i$ & / & $0.9802+0.0632 i$ \\
\hline & & 0.010 & $0.9668+0.1069 i$ & / & $0.9522+0.0973 i$ \\
\hline & & 0.015 & $0.9594+0.1599 i$ & / & $0.9510+0.1523 i$ \\
\hline & & 0.020 & $0.9492+0.2125 i$ & / & $0.9526+0.2208 i$ \\
\hline & \multirow{4}{*}{0.070} & 0.005 & $0.9704+0.0535 i$ & / & $0.9708+0.0673 i$ \\
\hline & & 0.010 & $0.9660+0.1068 i$ & / & $0.9500+0.1006 i$ \\
\hline & & 0.015 & $0.9586+0.1598 i$ & / & $0.9458+0.1549 i$ \\
\hline & & 0.020 & $0.9484+0.2123 i$ & / & $0.9328+0.2232 i$ \\
\hline \multirow{12}{*}{21 degrees } & \multirow{4}{*}{0.068} & 0.005 & $0.9720+0.0536 i$ & / & $0.9565+0.0686 i$ \\
\hline & & 0.010 & $0.9675+0.1070 i$ & / & $0.9230+0.1028 i$ \\
\hline & & 0.015 & $0.9602+0.1601 i$ & / & $0.9201+0.1575 i$ \\
\hline & & 0.020 & $0.9499+0.2126 i$ & / & $0.9250+0.2253 i$ \\
\hline & \multirow{4}{*}{0.069} & 0.005 & $0.9712+0.0535 i$ & / & $0.9432+0.0743 i$ \\
\hline & & 0.010 & $0.9668+0.1069 i$ & / & $0.9195+0.1075 i$ \\
\hline & & 0.015 & $0.9594+0.1599 i$ & / & $0.9122+0.1612 i$ \\
\hline & & 0.020 & $0.9492+0.2125 i$ & / & $0.8970+0.2288 i$ \\
\hline & \multirow{4}{*}{0.70} & 0.005 & $0.9704+0.0535 i$ & / & $0.9345+0.0780 i$ \\
\hline & & 0.010 & $0.9660+0.1068 i$ & I & $0.9168+0.1106 i$ \\
\hline & & 0.015 & $0.9586+0.1598 i$ & / & $0.9065+0.1637 i$ \\
\hline & & 0.020 & $0.9484+0.2123 i$ & / & $0.8783+0.2312 i$ \\
\hline
\end{tabular}

Using this linear regression for the $N=36$ points given in the table above (coordinates angle, radial position, $\mathrm{x}$-coordinate) we found the following currents for the best least squares 
fit, Ring 1 current $=0.00998+0.0673 i$ milliamperes; Ring 2 current $=-0.01883+0.0480 i$ milliamperes and respectively Ring 3 current $=-0.173-0.507 i$ milliamperes. The quality of the statistical fitting process can be examined by review of the table. This table lists the field values ("data") at the 36 points considered versus the values obtained by the regression model shown at the right of the data values.

We immediately notice that there are little or no differences in the field of the $T M_{01}$ mode between the angles of 19, 20 and 21 degrees. This implies that fitting can be accomplished over a larger volume segment. There are noticeable changes in the $T M_{01}$ field with respect to radial distance and with respect to position in the waveguide relative to the entire array as captured by the variable $x$ (smaller values of $x$ in meters correspond to positions nearer the left-most ring or sub-array of the full array. The statistical fit appears to track the change in the imaginary component of the actual field better than it tracks the real part.

The reader might question why our focus in the least squares or linear regression work centered on the real and imaginary parts of the fields rather than using the amplitude and phase. Our reasoning is that when measurements are contaminated with noise, as is the assumption in least squares or regression analysis, the noise enters into the real and imaginary parts of the Fourier transform of the spectral estimate as approximately independent components [6] and this is an important assumption in our least square analysis in the context of our simulation.

We measured the degree of fit using the standard error which is the ratio of the standard derivative of the variable divided by the mean of the variable. We have found that the standard error of the real part of the regression fit is $3.9 \%$ and the standard error of the imaginary part is $9.3 \%$. In terms of absolute errors of fit in the real and imaginary components, more variability in the fit of the real part is noted than of the phase as can be understood from the fact that the mean of the real part of the field points is in the neighborhood of one volt per meter, while the mean of the imaginary part of the field point is roughly 0.15 volts per meter.

We recognize that a linear regression analysis could fit chosen data points acceptably but fail at field points between the chosen points. Therefore, we tested our regression equation by computing test or check values at points that were not necessarily our chosen points in space. As shown in the Table above we fit our equation between the radial angles of 19-21 degrees, radial distance from 0.068 to 0.70 meters, and $\mathrm{x}$, the longitudinal distance, ranging from 0.005 to 0.025. In the figures below (Figure 8 and Figure 9) we compare the actual field value as computed using the mode equation with the predicted value at points that are generally different from those we use to fit the regression equation. We show these figures using the radial angle of 20 degrees, and at radius 0.069 meters. Figure 8 is of the real part of the field, and Figure 9 is of the imaginary part of the field. As in the above Table where the imaginary part of the field seemed better fitted, in this check the regression equation used as a predictive equation seemed to do a better job on the imaginary part.

What we have demonstrated with the above simulations is that, as predicted by the theoretical development, it is possible to approximately establish a predetermined field within a finite volume in front of an antenna in a cylinder in such a manner as to match a $T M_{01}$ mode as it exists in that limited volume. Thus, for example, locally the mode can be nulled or, if needed, the phase can be shifted by generating a signal with near equivalent amplitude 


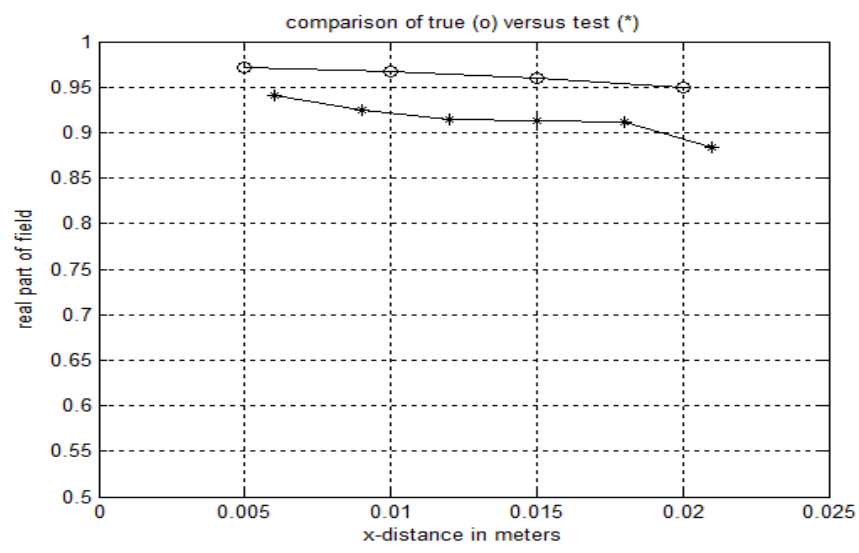

Figure 8: . Real part of the impressed field (TM $M_{01}$ mode) within the cylinder labelled with the o symbol. Labelled with the * symbol are the predicted values. The work was done at 20 degrees, and 0.069 meters within the cylinder.

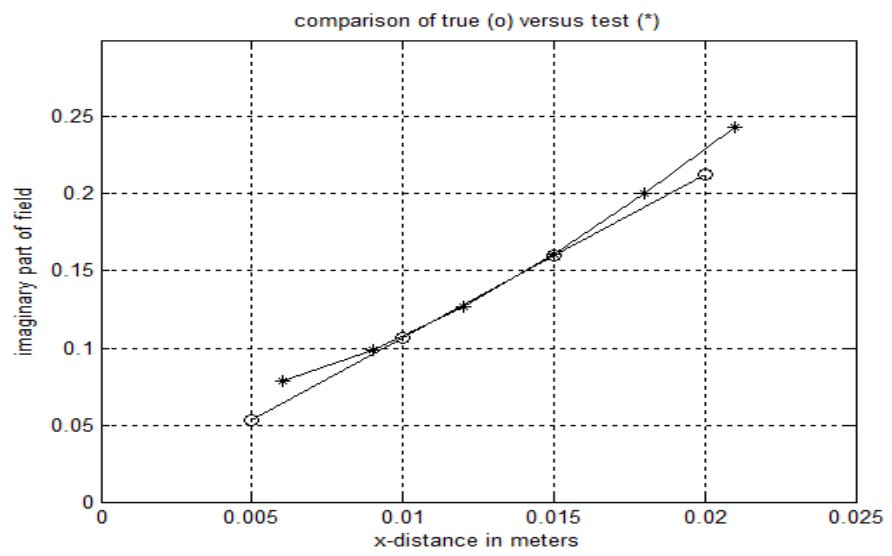

Figure 9: Imaginary part of the impressed field ( $T M_{01}$ mode) within the cylinder labelled with the o symbol. Labelled with the * symbol are the predicted values. The work was done at 20 degrees, and 0.069 meters within the cylinder.

but different phase.

Remark 3.2. Note that in our least square analysis we only considered the case when all the dipoles in a ring are driven by the same current, (i.e. six degrees of freedom, three amplitudes and three phases). At this point we believe that allowing more degrees of freedom (individual feed currents for each dipole) will improve our results.

As reflected in the introduction, we intuitively felt that an antenna system (for example, an array) could definitely form a field that was closely equivalent to an incoming field at one field point at least. We conjectured that an equivalence might be achieved on a line and if the incoming and array-produced fields are smooth, there may be nulling over a small volume sector. The theoretic development indicates that under the condition of field smoothness, a volumetric equivalence or nulling is possible, and this has been strongly suggested by the above reported numerical work. 
Remark 3.3. Due to the radial symmetry, based on our simulations, we make the observation that a similar array as the one considered above will provide the desired control results of the $T M_{0 n}$ modes.

\subsection{Method of Moments checks on the analytical array design}

To the best of our knowledge no one has ever used the field representation in [41] (see (3.30)) or any other similar analytical result to compute fields of dipoles within a cylindrical waveguide with conducting walls. Thus, we sought to check our work using a well-known wire based method of moments code 4NEC2.

Using $4 \mathrm{NEC} 2$, we simulated a 0.01 meter dipole driven by $573 \mathrm{Mhz}$ within a 3 meter long circular cylindrical waveguide centered at $x=1.5$ and with radius 0.5 meters. Note that the small dipole used in this work is smaller than the $1 / 50$-th of the wavelength so the $4 \mathrm{NEC} 2$ work should be comparable to the analytical work. The small dipole was placed, as was the infinitesimal dipole computed using the analysis in [41], at 0.06 meters on the z-axis of the coordinate system with $y=0$ and $x=1.6$ meters.

(We worked with the dipole at $x=1.6$ meters above the base of the waveguide since placing the dipole exactly on the central plane, $x=1.5$, led to discretization exceeding our computational capability: we computed every 0.2 meters in $\mathrm{x}$, while computing every 0.006 meters in the $\mathrm{y}$ and $\mathrm{z}$ axis.)

The longitudinal component of the field of this driven dipole within the waveguide is shown below in Figure 10 and the field pattern at 0.07 meters radial distance at $x=1.6$ meters is shown following in Figure 11. Notice that using 4NEC2 we can compute a pattern in a plane that included the center of the dipole, something we could not do with equation (3.30) due to convergence issues in this plane resulting from the residue analysis. It is interesting to us that the pattern in the plane of the center of the short dipole is similar to that computed with (3.30).

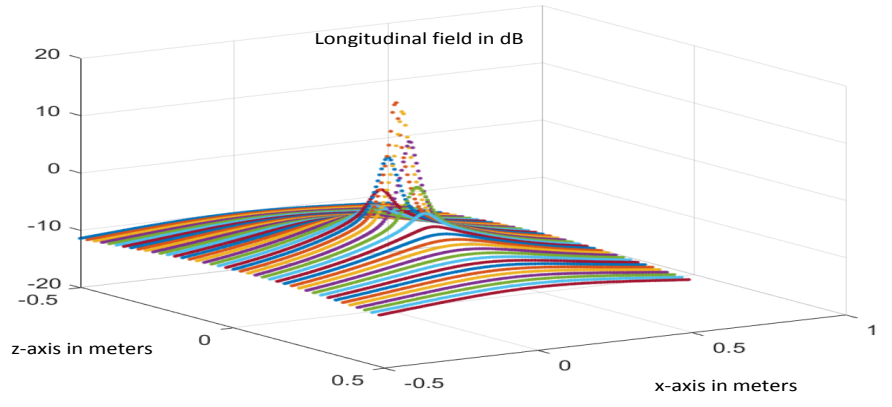

Figure 10: Longitudinal component of the field within a circular cylindrical waveguide of length 3 meters and diameter 0.05 meters. This plot of the overall pattern is in $d B$ units. The single dipole was one centimeter in length and was placed eccentrically at $(x, \varphi, r)=(1.6,0,0.06)$ and was driven by a $573 \mathrm{Mhz}$ sinusoidal voltage. The general shape of this field profile agrees with that computed using (3.30) 


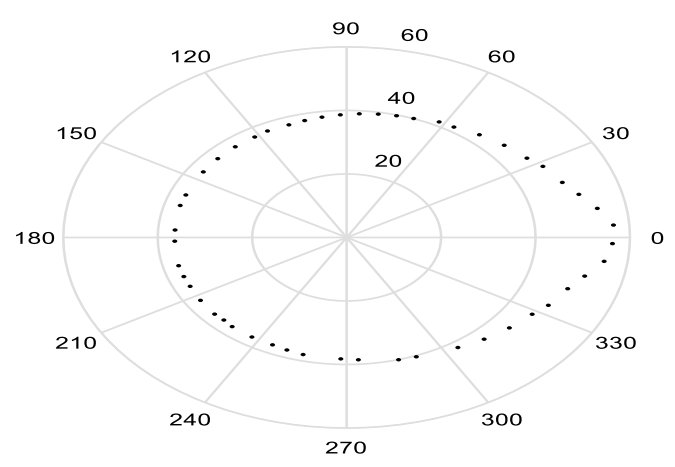

Figure 11: Longitudinal component of the field at a radial distance of 0.07 meters from a single dipole within a circular cylindrical waveguide of length three meters and diameter 0.05 meters. This plot of the radiation pattern is in $d B$ units. The single dipole is one centimeter in length and was placed eccentrically at $(x, \varphi, r)=(1.6,0,0.06)$ axis and was driven by a 573 Mhz sinusoidal voltage. The general shape of this field profile agrees with that computed using (3.30)

These numerical results using 4NEC2 provide some initial supportive results concerning our computations with the formulation in [41] (described in (3.30)). The method of moment method also allows us to consider practical issues of dipole impedance and coupling between dipoles.

The impedance of this small dipole inside the cylinder is computed by 4NEC2 as $2.21-$ 365i. The impedance of this dipole outside the cylinder (in free space) was computed as $2.58-363 i$. As expected, the impedance of these small dipoles is very low. However, it is important to see that in this case the impedance is not greatly affected by placement into the waveguide. In practice, the low impedance will lead to mismatch problems with respect to the signal source, however, the antennas themselves can be loaded to mitigate this issue, we believe.

We also address the coupling issue in a preliminary or initial fashion. Using 4NEC2 again we simulated two parallel dipoles 0.01 meters long and 0.03 meters apart. The 0.03 meter distance is relevant to our sub-array ring structures. Impedance of the dual dipole in free space is computed to be $4.77-385 i$ and in the cylinder, with one dipole at 0.03 meters and the second at 0.06 meters, to be $4.25-389 i$. Thus, there is coupling and the result is an increase in input impedance compared to the single dipole but, based on our simulations the effect does not strikingly depend on placement within the cylinder. We expect this change between free space and within the cylinder to increase as we consider smaller diameter cylinders, but we leave this for future work.

It was recommended that we look still closer at dipole-dipole coupling within the cylinder comparing this coupling within the cylinder to coupling of the same two dipoles outside in free space. In free space, application of a one volt peak sinusoidal voltage to the central segment of the dipole at 0.06 meter resulted in a current of $2.77 \mathrm{e}-3$ amperes with phase 89.55 degrees in the driven dipole. This driven dipole caused $1.78 \mathrm{e}-4$ ampere on the central segment of the parallel second dipole at 0.03 meters with phase -84.89 degrees. Within the cylinder, current on the central segment of the excited dipole at 0.06 meters was 2.05e-3 
amperes (phase 89.62 degrees) and current on the central segment of the parallel coupled dipole at 0.03 meters was computed to be $1.91 \mathrm{e}-4$ with phase -85.67 degrees. Thus, we conclude that coupling between the dipoles is enhanced by placement within the cylinder, but at this time, we do not perceive that coupling between dipoles will be a limiting factor.

In considering the numerical work that has been done, we believe the observation that we can control a small volume in front of the idealized array is made possible by the fact that we are using small radiating antennas relative to wavelength and thus while there is a significant near field, there is little radiated field. At this time we believe the greatest part of the nulling activity in our numerical work is accomplished by fields that remain attached to the antennas (near field).

\subsection{Slot antennas and arrays}

The mathematical argument of Section 2.2 provides an analytical scheme whereby fields can be controlled within a circular cylindrical waveguide using electrically active surfaces included within the guide. Section 3 has provided numerical results on a realization of such a system to provide local control of a $\mathrm{TM}_{01}$ mode.

We now briefly discuss one of the reasons we are interested in this theory and numerical result. We believe there may be application to slot antennas.

A radiating slot is simply a slit in a conducting surface driven by a voltage across the slit as diagrammed in Figure 12 obtained from [1]. For a slot to radiate it must cut current lines in the homogeneous surface (the surface without the slot). In the circular cylindrical waveguide the currents associated with a $T M_{01}$ mode are axial and one may use axially placed slots in the waveguide so that the radiation produced is directly related to the width of the slot. A slot array is comprised of a series of slots placed along the waveguide; slots in such a surface will couple (share energy) electromagnetically. Slot antennas are attractive

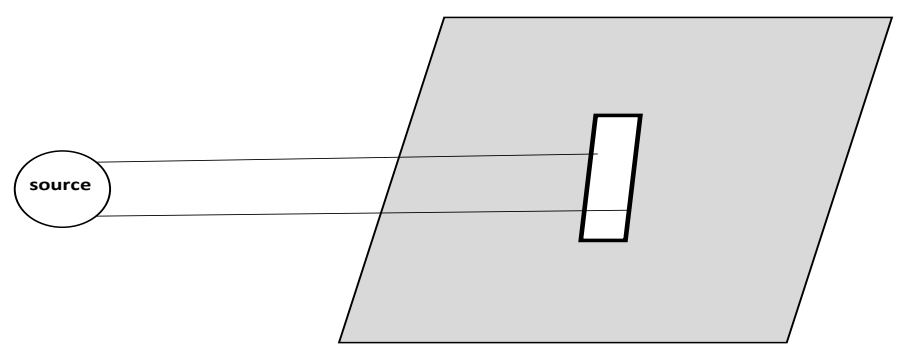

Figure 12: Schematics of a radiating slot: Rectangular slit in a grey conducting surface driven by a voltage across the slit (courtesy of [1]).

for use on aircraft or other airborne vehicles since they can be incorporated into structure surfaces with minimal aerodynamic effects [3]. In [27] the authors report on annular slot antennas driven by waveguide modes. Non-mechanical pointing of a slot array requires a change in frequency because of the coupling between slots [52].

The concept being pursued by us is to use a local array, as sketched above, to adjust the phase of the field driving a slot so that the desired pointing can be achieved without 
change of frequency. We have some support for this concept because of the successful use of magnetized ferrite materials in slot arrays [24]. We have been most encouraged in this work by the work in [28] which addresses the slot antenna associated with two coupled dipoles. This paper, important to our thinking, studies the effect of unexcited, parasitic (passive) dipoles on either side of a single slot and effects on radiation pattern studied.

A slot cutting a surface current, can be qualitatively considered as two short dipoles at each end of the slot parallel to the current path [28] (pp. 143). Thus, $n$ additional dipoles, parallel to these edges effectively compose an array with $n+2$ elements and this facilitates beam control. Figure 13 shows a diagram illustrating the case of one additional dipole behind the slot inside the waveguide. Computation with 4NEC2 has indicated that driving the backing dipole influences the phase of the radiated field and thus may assist in beam steering in a slot array structure. Our investigation on this application will be continued and results reported in a forthcoming paper.

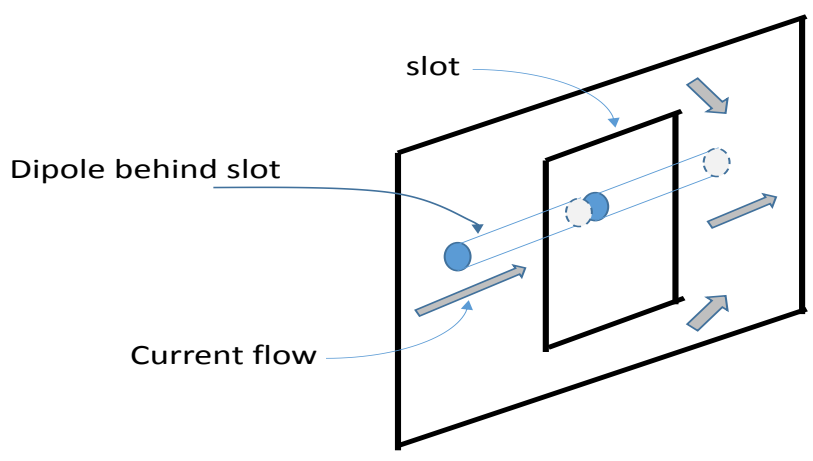

Figure 13: Configuration of a single dipole behind a slot. Computation with 4NEC2 indicates that the dipole, driven at the same frequency as the slot, can influence the phase of the far field radiation thus potentially assisting in phased array beam steering.

\section{Summary and Comments}

If an arbitrary field is launched from a source at a second antenna driven by a field of the same frequency with amplitude and phase randomly chosen, one would not expect that the source and target antenna field would necessarily anywhere destructively interfere. This is clear by the fact that the source strength can simply be chosen very much higher so that it dominates the field of the second antenna. However, it seems clear that for an incident plane wave and a suitable array the two fields can destructively interfere exactly at a point and perhaps along a short tubular segment with small radius, assuming miniscule difference between the incoming source field and the field of the second antenna. In this paper, a mathematical argument has been advanced that within a circular cylindrical waveguide with perfectly conducting walls, a radiating source internal to the waveguide can destructively interfere with a waveguide mode in a finite annular volume near the radiating source and the destructive interference can occur with small differences noted between the waveguide mode 
and the field radiated by the internal antenna. This mathematical proof is not such that the defined field can be directly computed at this time, but the proof has been numerical tested using simulation. In the numerical work a small 24 element circular array composed of three ring subarrays of eight dipoles each has been used to match a $T M_{01}$ mode and this matching, while not exact, has shown approximate field matching especially in the complex component of the field. Each dipole in each subarray received the same current and these currents have been estimated. We believe, at this time, that better field matching can be accomplished by either using more subarrays or driving each dipole in each subarray separately. This study was motivated by a desire to begin to mathematically understand how antenna currents can be chosen to destructively interfere with incoming fields with a view, ultimately, to applications such a beam steering in cylindrical slot arrays.

\section{Acknowledgment}

This work was initially funded by the AFOSR under the award FA9550-13-1-0078 and subsequently by ONR under the award N00014-15-1-2462.

\section{References}

[1] J. S. Ajioka, On the Conceptual Utility of Current Flow Lines in Waveguides. IEEE Trans. In Antennas and propagation, Vol. 34., Iss. 2, pp. 219-227, 1986.

[2] A. Alford, R. Sprague, A four slot cylindrical antenna for VOR service, IRE International Convention Record, Vol.2, pp.12-24, 1954.

[3] L. L. Bailin, Radiation Field Produced by a Slot in a Large Circular Cylinder, IRE Transactions Antennas and Propagation, Vol.3, Iss. 3, pp. 128-137, 1955.

[4] A. Bakushinsky, A. Goncharsky, Ill-Posed Problems: Theory and Applications, Kluwer Academic Publishers, 1994.

[5] C. A. Balanis, Antenna Theory; Analysis and Design, John Wiley and Sons, New York, 2005.

[6] D. R. Brillinger, Time Series: Data Analysis and Theory, Holt, Rinehardt and Winston, INc, New York, pp. 95-96, 1975.

[7] D. Colton and R. Kress, Integral Equation Methods in scattering Theory, Pure \& Applied Math., Wiley Interscience, New York, 1983.

[8] G T Clement, K Hynynen, A non-invasive method for focusing ultrasound through the human skull, Phys. Med. Biol. 47, pp. 12191236, 2002.

[9] P. Deo, D. Mirshekar-Syahkal, L. Seddon, S. E. Day, F. Anbal Fernndez, Beam Steering $60 \mathrm{GHz}$ Slot Antenna Array using Liquid Crystal Phase Shifter, IEEE report of The 8th European Conference on Antennas and Propagation (EuCAP), pp. 1005-1007, 2014. 
[10] J. Du, S. Liu, Z. Lin, Broadband Optical Cloak and Illusion Created by the Low Order Active Sources, Opt Express., Apr 9, Vol. 20, Iss. 8, pp. 8608-8617, 2012.

[11] G. V. Eleftheriades, M. Selvanayagam, An Active Electromagnetic Cloak Using the Equivalence Principle, IEEE Ant. and Wireless Prop. Lett., Vol. 11, pp. 1226-1229, 2012.

[12] G. V. Eleftheriades, M. Selvanayagam, Experimental Demonstration of Active Electromaagnetic Cloaking, Phys. Rev. X, vol. 3, Iss. 4, pp. 041011-1, 041011-13, 2013.

[13] S.J. Elliot, P.A. Nelson, The active control of sound, Electronics and Comm. Engineering Journal, August, 1990.

[14] W.C. Elmore, M.A. Heald, Physics of waves, Dover Publications Inc., New York, 1985.

[15] H. W. Engl, M. Hanke, A. Neubauer, Regularization of Inverse Problems, Springer Science \& Business Media, 1996.

[16] C.R. Fuller, A.H. von Flotow, Active control of sound and vibration, IEEE Contr. Systems, Vol. 15, Iss. 6, pp. 9-19, 1995.

[17] R. Garg, K. C. Gupta, Suppression of Slot Mode in Slotted Waveguide Antennas, IEEE Transactions on Antennas and Propagation, pages 731-732, 1975.

[18] F. Guevara Vasquez, G. W. Milton, D. Onofrei, P. Seppecher, Transformation elastodynamics and active exterior acoustic cloaking, Acoustic Metamaterials, Springer Series in Materials Science, Vol. 166, pp. 289-318, 2012 (online at arXiv:1105.1221).

[19] F. Guevara-Vasquez, G.W. Milton, D. Onofrei, Mathematical analysis of the active exterior cloak for 2D quasistatic electromagnetics, Analysis and Mathematical Physics, Vol. 2, Iss. 3, pp. 231-246, 2012.

[20] F. Guevara Vasquez, G. W. Milton, and D. Onofrei, Active exterior cloaking, Phys. Rev. Lett., Vol. 103, Iss. 7, pp. 073901, 2009.

[21] — Broadband exterior cloaking, Optics Express, Vol. 17, pp. 14800-14805, 2009.

[22] — Exterior cloaking with active sources in two dimensional acoustics, Wave Motion, Vol. 48, Iss. 6, pp. 515-524, 2011.

[23] A. Harmouch, H. Haddad, Cylindrical Omnidirectional Slotted Waveguide Antenna with Optimized Directional Characteristics, 13th Mediterranean Microwave Symposium, pp. 1-4, Sept. 2013.

[24] K. C. Hwang, J. E. Hyo, Slot array antenna using a rectangular waveguide partially filled with a ferrite slab, Proc. IEEE Int. Workshop Antenna Tech. Small Antennas Novel Metamaterials, pp 412-415, 2006. 
[25] C. Q. Jiao, Selective Suppression Of Electromagnetic Modes In A Rectangular Waveguide By Using Distributed Wall Losses, Progress In Electromagnetics Research Letters, Vol. 22, pp. 119-128, 2011.

[26] M. F. Jamlos, O. A. Aziz, T. A. Rahman, M. R. Kamarudin, P. Saad, M. T. Ali, M. N. Md Tan, A Beam Steering Radial Line Slot Array (RLSA) Antenna with Reconfigurable Operating Frequency, J. of Electromagn. Waves and Appl., Vol. 24, Iss. 8-9, pp. 1079 1088, 2010.

[27] K. C. Kelley, F. J. Goebels, Annular Slot Monopulse Antenna Arrays, IEEE Trans on Antennas and Propagation, Vol. 12, Iss. 4, pp. 391-403, 1964.

[28] R. W. P. King, G. H. Owyang, The Slot Antenna with Coupled Dipoles, IRE Trans Antennas and Propagation, pp 136-143, March 1960.

[29] J. E. Lagnese, Exact Boundary Controllability of Maxwell's equations in a general region, Siam Jour. of Contr. and Opt., Vol. 27, Iss. 2, pp. 374-388, 1989.

[30] J. E. Lagnese, G. Leugering, Dynamic Domain Decomposition in Approximate and Exact Bounday Control in Problems of Transimission for Wave Equations, Siam Jour. of Contr. and Opt., Vol. 38, Iss. 2, pp. 503537, 2000.

[31] J. E. Lagnese, G. Leugering, Domain Decomposition in Optimal Control of Partial Differential Equations, Birkhauser, 2004.

[32] K.-S. Lim, V.-C. Koo, T.-S. Lim, Design, Simulation and Measurement of a Post Slot Waveguide Antenna, J. of Electromagn. Waves and Appl., Vol. 21, Iss. 12, pp.15891603, 2007.

[33] C. L. Law and E. K. N. Yung, A Cylindrical Slot Waveguide Antenna, IEEE report of Asia-Pacific Microwave Conference, Adelaide, Vol.1, pp. 133-136, 1992.

[34] P. Leug, Process of silencing sound oscillations, U.S. patent no. 2043416, 1936.

[35] J. Loncaric, V.S. Ryaben'kii, S.V. Tsynkov, Active shielding and control of environmental noise, technical report, NASA/CR-2000-209862, ICASE Report No. 2000-9,online at: http://ntrs.nasa.gov/search.jsp?R=20020075003, 200

[36] Q. Ma, Z. L. Mei, S. K. Zhu, and T. Y. Jin, Experiments on Active Cloaking and Illusion for Laplace Equation, Phys. Rev. Lett., vol. 111, Iss. 17, pp. 173901, 2013.

[37] K. Martiny, M. Lunde, P. Bech, Transcranial Low Voltage Pulsed Electromagnetic Fields in Patients with Treatment-Resistant Depression, Biol. Psychiatry, Vol.68, Iss.2, pp. 163-169, 2010.

[38] D. A. B. Miller, On perfect cloaking, Opt. Express, Vol. 14, pp. 12457-12466, 2006.

[39] K. S. Miller, Complex Stochastic Processes; An Introduction to Theory and Application, Addison-Wesley Publishing Company, Inc., 1974. 
[40] C. Muller, Foundations of the Mathematical Theory of Electromagnetic waves, Springer-Verlag, 1969.

[41] C. Muzikar, Electromagnetic Waves Excited by an Elementary Electric Dipole in a Cylindrical Waveguide, Czechoslovak Journal of Physics, Vol. 1, pp. 127-133, 1952.

[42] A. N. Norris, F. A. Amirkulova, W. J. Parnell, Source amplitudes for active exterior cloaking, Inverse Problems, Vol. 28, Iss. 10, 2012.

[43] H.F. Olson, E.G. May, Electronic sound absorber, J. Acad. Soc. America, Vol. 25, pp. 1130-1136, 1953.

[44] D.Onofrei, On the active manipulation of fields modeled by the Helmholtz equation., Journal Of Integral Equations and Applications, Vol. 26, Iss. 4, pp. 553-579, 2014.

[45] D. Onofrei, On the active manipulation of fields and applications. I - The Quasistatic regime, Inverse problems, Inverse Problems Vol. 28, No. 10, 2012.

[46] N. Peake, D.G. Crighton, Active control of sound, Annu. Rev. Fluid Mech., Vol.32, 137-164, 2000.

[47] A.W. Peterson, S.V. Tsynkov, Active control of sound for composite regions, SIAM J. Appl. Math, Vol. 67, Iss. 6, pp. 1582-1609, 2007.

[48] V.S.Ryaben'kii, S.V.Tsynkov, S.V.Utyuzhnikov, Active control of sound with variable degree of cancellation, Applied Mathematics Letters, Vol. 22, pp. 1846-1851, 2009.

[49] S. A. Schelkunoff, Electromagnetic Waves, D. Van Nostrand Company, Inc., New York, 1943.

[50] G. Thamsborg, A. Florescu, P. Oturai, E. Fallentin, K. Tritsaris, S. Dissing, Treatment of knee osteoarthritis with pulsed electromagnetic fields: a randomized, double-blind, placebo-controlled study, Osteo Arthritis and Cartilage, Vol. 13, Iss. 17, 575-581, 2005.

[51] M. C. J. Trinder, P. A. Nelson, Active Noise Control in Finite Length Ducts, Journal of Sound and Vibration, Vol. 89, Iss. 1, pp. 95-105, 1983.

[52] H. J. Visser, Array and Phased Array Antenna Basics, John Wiley \& Sons, Ltd, pp 223-224, 2005.

[53] R. Weyrich, Uber einige Randwertprobleme, insbesondere der Elektrodynamik, Journal fur die reine und angewandte Mathematik, Vol. 172,pp. 133-150, 1935.

[54] P. Wounchoum, C. Phongcharoenpanich, D. Worasawate, M. Krairiksh, Radiation Characteristics of Two-Slot Array Antenna on a Concentric Sectoral Cylindrical Resonator Excited by a Coupling Slot, IEEE report of Proceedings of Asia-Pacific Microwave Conference, pp. 1-4, December 2007.

[55] H.H. Zheng, J.J.Xiao, Y. Lai, C.T. Chan, Exterior Optical Cloaking and Illusions by Using Active Sources: A Boundary Element Perspective, Phys. Rev. B, Vol., 81, Iss. 19, pp. 195116-195127, 2010. 